\title{
Quantitative Assessment of Radiation-nduced Changes in the Connective Tissue Matrix of the Urinary Bladder by Nonlinear Microscopy
}

DOI: $10,17691 / \mathrm{stm} 2018,10.3 .14$

Received January 18, 2018

V.V. Dudenkova, Junior Researcher, Scientific Laboratory of Optical Coherence Tomography,

Institute of Biomedical Technologies ${ }^{1}$; Assistant, Department of General Physics, Faculty of Radiophysics;

A.V. Maslennikova, MD, DSc, Professor, Oncology, Radiotherapy, Radiodiagnosis Department ${ }^{1}$; Professor, Department of Biophysics;

E.B. Kiseleva,PhD, Researcher, Scientific Laboratory of Optical Coherence Tomography, Institute of Biomedical Technologies ${ }^{1}$;

E.A. Tararova, MD, PhD, Oncologist ${ }^{3}$;

K.E. Yunusova, MD, PhD, Associate Professor, Department of Pathological Anatomy ${ }^{1}$;

O.S. Streltsova, MD, DSc, Professor, Urology Department named after E.V. Shakhov ${ }^{1}$

${ }^{1}$ Privolzhsky Research Medical University, 10/1 Minin and Pozharsky Square, Nizhny Novgorod, 603005, Russia;

2Lobachevsky State University of Nizhni Novgorod, 23 Prospekt Gagarina, Nizhny Novgorod, 603950, Russia;

${ }^{3}$ Nizhny Novgorod Regional Oncologic Hospital, 190 Rodionova St., Nizhny Novgorod, 603126, Russia

The aim of the investigation was to study the possibilities of the quantitative assessment of the bladder collagen and elastin state using nonlinear microscopy for determining the degree of side effect severity after the radiotherapy of female reproductive system tumors.

Materials and Methods. The object of investigation was a biopsy material from nine patients undergone radiotherapy for malignant neoplasms of the small pelvis organs in their past history, and from three patients treated for chronic cystitis of bacterial etiology. The extracellular matrix of the bladder connective tissue was examined by nonlinear microscopy in the modes of the second harmonic generation (SHG) and two-photon excited autofluorescence (TPEF) on $10 \mu \mathrm{m}$ unstained dewaxed histological sections. 133 urinary bladder images of the patients divided into 4 groups according to their clinical picture (104 images with the radiation damage grade II $(n=24)$, III $(n=40)$, and IV $(n=40)$ ) were quantitatively and qualitatively analyzed and compared with 29 bladder images of the patients with chronic cystitis.

Mean value of intensity and a non-uniformity parameter of SHG and TPEF signals were chosen as indices of the quantitative assessment (collagen and elastin state in terms of their form and packing density, integrity/disorganization, and orientation).

Results. In the given bladder pathology, the state of the collagen and elastin fibers grows from radiation cystitis grade II to grade IV while in chronic cystitis the picture may be similar with radiation cystitis grade II or III alike. Alterations in the collagen and elastin fibers with the loss of the native structure and spatial arrangement are seen on part of the images as a fuzzy fiber contour (blurring), disorganization/ fragmentation, areas with a dense random fiber arrangement or anhistic areas with a high signal.

Mean value of collagen SHG and elastin TPEF signal intensity in severity grade II was statistically significantly higher than in grades III and IV $(p \leq 0.05)$. Such decrease of the values reflects a marked disorganization (fragmentation) of the fibers in comparison with their native structure which results in the lower ability of the fibers to generate SHG or TPEF signal. Increase of the SHG signal non-uniformity values in grade III and IV radiation damage of the urinary bladder compared to grade II and chronic cystitis reflects the same tendency.

Conclusion. A nonlinear microscopy method makes it possible to assess both qualitatively and quantitatively spatial and structural organization of the extracellular bladder matrix in radiation damage and chronic cystitis, to determine the severity degree of complications in radiation damage, and to predict their course.

Key words: extracellular matrix; urinary bladder; radiotherapy; radiation damage of the urinary bladder; chronic cystitis; nonlinear microscopy; collagen; elastin.

\section{Introduction}

In recent years, pelvic organs in malignant neoplasms of the female reproductive system are treated by conformal irradiation methods enabling the radiation dose to be maximally concentrated on the target with a high gradient relative to the surrounding nonneoplastic tissues. Nevertheless, the side effects of irradiation relative to the urinary bladder and rectum, in particular, remain the unsolved problem in current radiation oncology [1]. The severity degree of side-effects from radiation/chemoradiation therapy can vary from

Corresponding author: Olga S. Streltsova, e-mail: strelzova_uro@mail.ru 
insignificant functional disorders to heavy damages affecting essentially patients' quality of life and resulting in disability [2, 3].

The pathogenesis of radiation-induced damages of normal tissues is complex and includes the interaction of a great number of tissue and cellular factors [4], vascular reaction development [5], and progressing changes in extracellular matrix as well $[6,7]$. The latter is especially important since it is the processes of the connective tissue stroma degradation that determines the development of III-IV grade side effects (bleeding, necrosis, fistula) which reduce dramatically patient's quality of life and are life-threatening [8].

In this situation, a question of precise quantitative assessment of radiation-induced changes in the extracellular matrix of normal tissues is of especial actuality. The current assessment systems (LENT/ SOMA, RTOG/EORTC, CTCAE) are mainly based on patients' complaints and visual examination data [9]. The development of optical techniques, e.g. optical coherent tomography (OCT) and multiphoton microscopy, allowing the assessment of the extracellular matrix at the tissue or cellular level both ex vivo $[10,11]$ and in vivo [12, 13], opens new potentialities for studying and classifying radiation side effects. Exploration of the collagen state on the model medium at various levels of its hierarchical organization with the help of optical and physico-chemical methods enables the researchers to detect the main mechanisms of its degradation and subsequent remodeling after ionizing radiation [14]. The previous investigations carried out by means of crosspolarization OCT showed that in patients with early radiation responses of the urinary bladder, the damage occurs primarily in the zone of suburothelial connective tissue structures [15]. For the first time, nonlinear microscopy method in the modes of signal generation of the second harmonic (SHG) and two-photon excited autofluorescence (TPEF) used for the study of the bladder radiation damage provided the possibility to obtain the qualitative characteristics of the connective tissue matrix state in case of the side effects of various severity degrees [16]. A leading world trend of using nonlinear microscopy for studying the connective tissue matrix state consists in the transition to the quantitative assessment of images which permits the investigators to avoid the shortcomings of subjective visual evaluation [17] and to systematize and differentiate the clinical material as well [18].

The aim of the study was to assess quantitatively the bladder collagen and elastin state using nonlinear microscopy for determining the degree of side effects severity after the radiotherapy of female reproductive system tumors.

\section{Materials and Methods}

The object of investigation was a biopsy material from nine patients (three patients with radiation damages in each of clinical II, III, and IV grades) having in the medical history radiation therapy for malignant neoplasms of the pelvic organs from 1.5 to 20 years ago, and from three patients treated for chronic cystitis of bacterial etiology. The high risk of heavy radiationinduced damages of the pelvic organs is known [19] to have patients with chronic inflammatory diseases of the urinary bladder, therefore, patients with chronic cystitis in the given study served as a control group.

The work was carried out in compliance with the Declaration of Helsinky (2013). Clinical investigations in the bladder radiation damage were approved by the Ethics Committee of Privolzhsky Research Medical University. Informed consent for its conduction was obtained from all examined patients.

The biopsy material was collected from the bottom area of the bladder, "a hot zone" maximally subjected to radiation exposure, using a resectoscope (Karl Storz, Germany).

The state of the bladder connective tissue matrix was examined by nonlinear microscopy in SHG and TPEF modes on $10 \mu \mathrm{m}$ unstained dewaxed histological sections. LSM 510 META laser confocal scanning microscope (Carl Zeiss, Germany) with a selected $800 \mathrm{~nm}$ wavelength and $\sim 4 \mathrm{~mW}$ power was used in the work. MAI TAl femtosecond Ti:Sa laser (Spectra Physics, USA) with $\sim 80 \mathrm{MHz}$ pulse repetition rate and $100 \mathrm{fs}$ pulse duration was used for exciting radiation.

The detection was performed in two channels simultaneously using filters in the ranges of $362-415 \mathrm{~nm}$ (SHG signal for the collagen, green color in the images) and 480-554 nm (TPEF signal for the elastin, red color in the images). This approach allowed visualization of the elastin and collagen fiber structure of the unstained specimens. Fine structure images were built using PlanNeofluar oil immersion objective (Carl Zeiss, Germany) with $40 \times$ magnification and 1.3 numerical aperture which allowed us to obtain a $318 \times 318 \mu \mathrm{m}$ field of view.

133 urinary bladder images of the patients divided into 4 groups according to their clinical picture (104 images with the radiation damage grade II $(n=24)$, III $(n=40)$, and IV $(n=40)$ ) were quantitatively and qualitatively analyzed and compared with 29 bladder images of the patients with chronic cystitis. To verify the nonlinear microscopy images, the concurrently obtained histological sections stained with hematoxylin and eosin according to the standard procedure were studied. Histological specimens were examined using Leica DMLS binocular microscope (Leica, Germany).

The quantitative assessment of SHG/TPEF signal in the images was done using Image J program $(\mathrm{NIH}$, USA) [17]. Mean intensity value and a non-uniformity parameter of SHG and TPEF signals were chosen as indices of the quantitative assessment (collagen and elastin state in terms of their form and packing density, integrity/disorganization, and orientation). The mean value of the intensity reflects a relative number of collagen/elastin fibers in a unit of field of view, the 

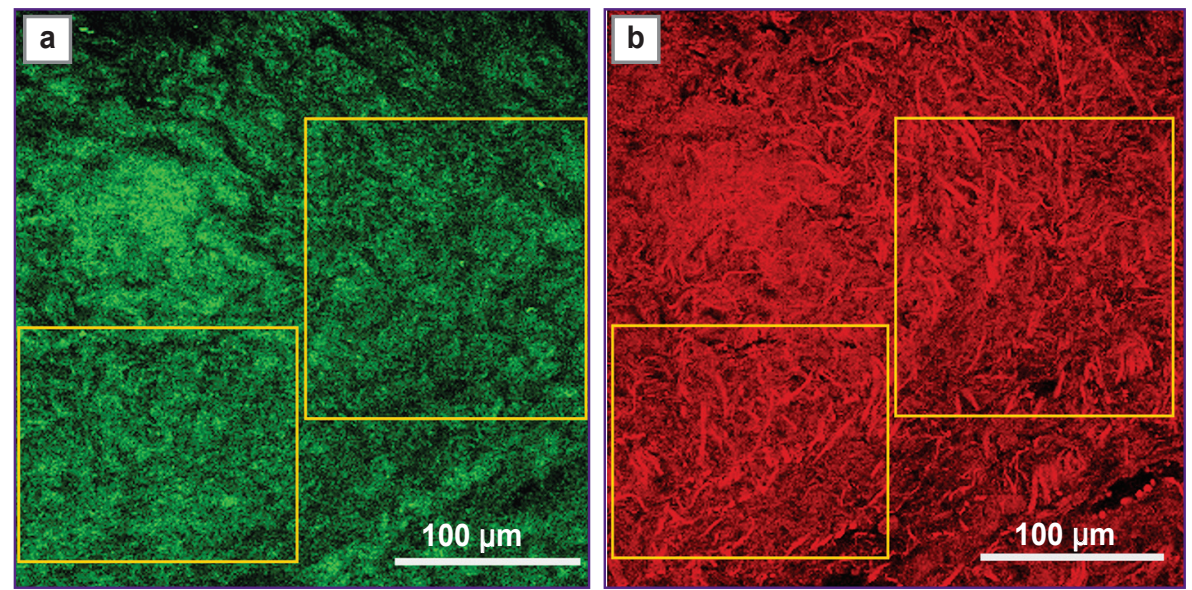

Figure 1. Example of regions-of-interest isolation used to assess quantitatively the state of collagen (a) and elastin (b) fibers of the connective tissue matrix form of fibers and the character of their packaging (a detail pathomorphological analysis of the images has been carried out by our research group in work [15]). In this work it was established that in the given bladder pathology, the state of the collagen and elastin fibers grows from radiation cystitis grade II to IV (Figure 2 (b)-(d)) while in chronic cystitis (Figure 2 (a)) the picture may be similar with radiation cystitis grade II or III alike. Alterations in the collagen and elastin fibers with the loss of the native structure and spatial arrangement are seen on part of the images

non-uniformity parameter characterizes the extent of fiber integrity in the field of view. The non-uniformity parameter was calculated as a ratio of standard deviation of the signal mean intensity over the selected area to a value of the mean intensity [18].

For the quantitative assessment of the stroma state, rectangular regions having typical tissue structure were chosen in the images of SHG and TPEF of the collagen and elastin. Not less than two regions of interest were marked out in each image (Figure 1). Indices of mean intensity and non-uniformity of SHG and TPEF were calculated by averaging the values obtained for the regions-of-interest set of the examined specimen. To evaluate the parameter of SHG signal non-uniformity, standard functions of computing the mean value and standard deviation over a selected region and a subsequent calculation of the latter/former ratio for each counted region were done [17].

Quantitative characteristics of SHG and TPEF signals between the chosen groups were compared depending on the pathological state (the degree of the radiation damage severity, chronic cystitis), which allowed us to detect radiation-induced alterations of the collagen and elastin structure and to correlate them with the severity of clinical manifestations of the radiation-damaged bladder tissue.

The data were statistically processed using Statistica 10.0 software package. In each group, the parameters of SHG and TPEF signals were characterized by mean and standard deviation $(M \pm S D)$. To compare the quantitative data of the two independent groups, the Student t-test was used. The differences were considered statistically significant at $p<0.05$.

\section{Results}

Images in the SHG and TPEF modes typical for each analyzed group are presented in Figure 2. Visual comparison of the images shows the differences in the fragmentation, areas with a dense random fiber arrangement (Figure $2(\mathrm{a})-(\mathrm{d})$ ), or anhistic areas with a high signal (Figure $2(\mathrm{~d})$ ). The structural alterations of the connective tissue matrix are supposed by us to be one of the causes of functional disorders of the bladder and are directly connected with the intensity of the clinical disease manifestations.

The results of the quantitative assessment of the SHG and TPEF images are given in Figure 3. The mean intensity value of the collagen SHG signal in severity grade II (124.8 \pm 21.0 standard unit) was statistically significantly higher than in grades III and IV $(87.6 \pm 10.3$ and $81.3 \pm 3.2$, respectively; $p \leq 0.05$ ) (Figure $3(a)$ ). The non-uniformity parameter dispersion of the collagen SHG signal in grades III and IV was statistically significantly higher than in grade II and chronic cystitis (in grades III and IV $-0.40 \pm 0.03$ and $0.45 \pm 0.09$, respectively; in grade $\|-0.29 \pm 0.05$ and in chronic cystitis $-0.28 \pm 0.01 ; p \leq 0.05)$ which is the evidence of a more marked impairment of the structural collagen package (Figure 3 (b)). In case of vesicovaginal fistula in grade IV damages, biopsy was performed from the lateral walls of the bladder, therefore the tissue changes were less expressed and corresponded to the alterations similar to the radiation damage grade II.

The mean intensity value of the elastin TPEF signal in severity grade II $(142.3 \pm 17.4)$ was statistically significantly higher than in grades III and IV (110.4 \pm 12.3 and $81.3 \pm 3.2$, respectively) (Figure 3 (c)). The dispersion of this parameter is less pronounced and has no statistically significant difference.

Values of the non-uniformity parameter distribution of the elastin TPEF signal in all groups do not differ statistically significantly $(p \geq 0.05)$ which may be interpreted as the evidence of a greater resistance of elastin to the radiation damage. This fact requires further exploration.

Thus, in grades III and IV of the bladder radiation as a fuzzy fiber contour (blurring), disorganization/ 


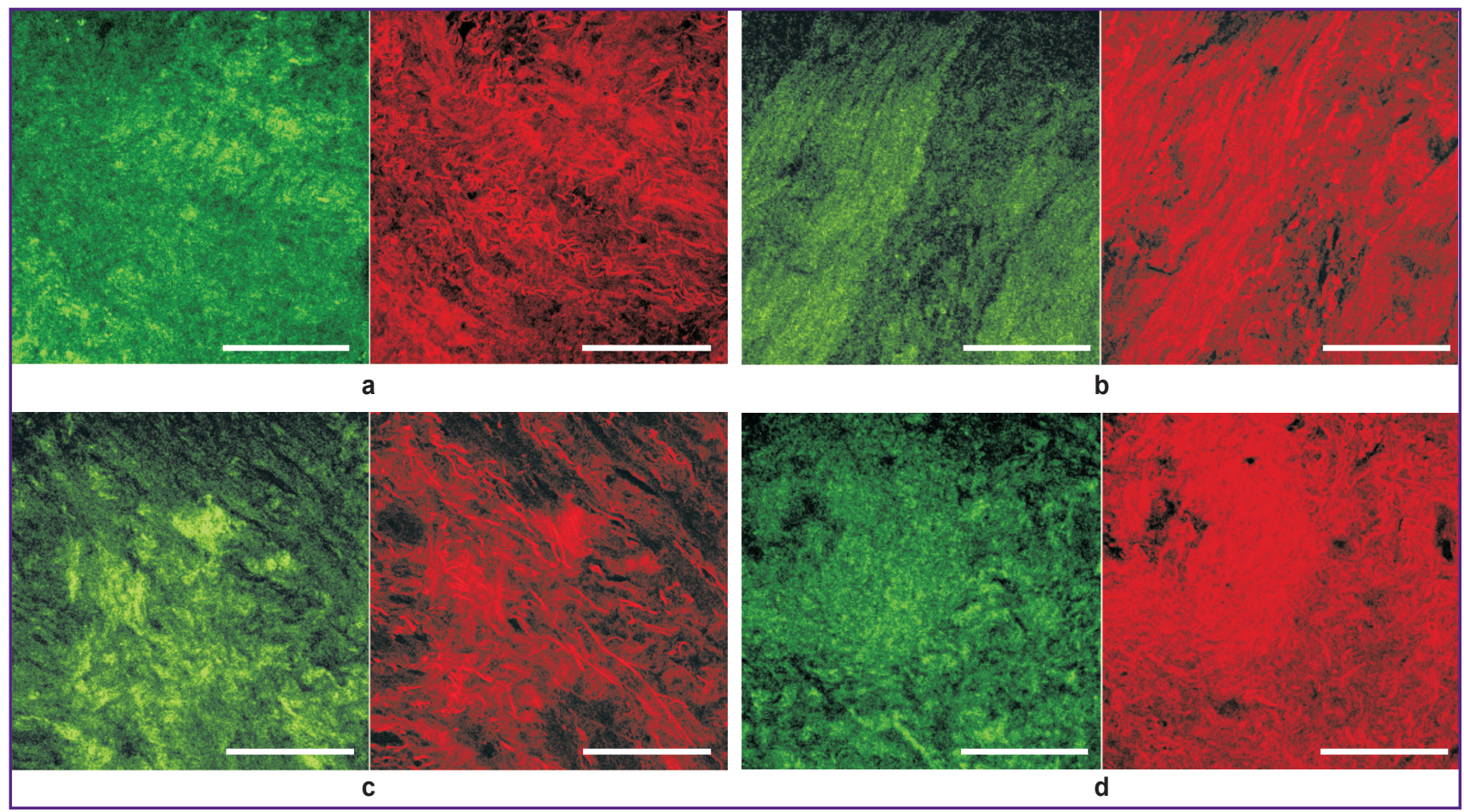

Figure 2. Images of the urinary bladder mucosa obtained by nonlinear microscopy:

(a) chronic cystitis; (b)-(d) complications of severity grades II, III, and IV after radiotherapy, respectively; in each pair of images, SHG is on the left, TPEF — on the right; bar - $100 \mu \mathrm{m}$
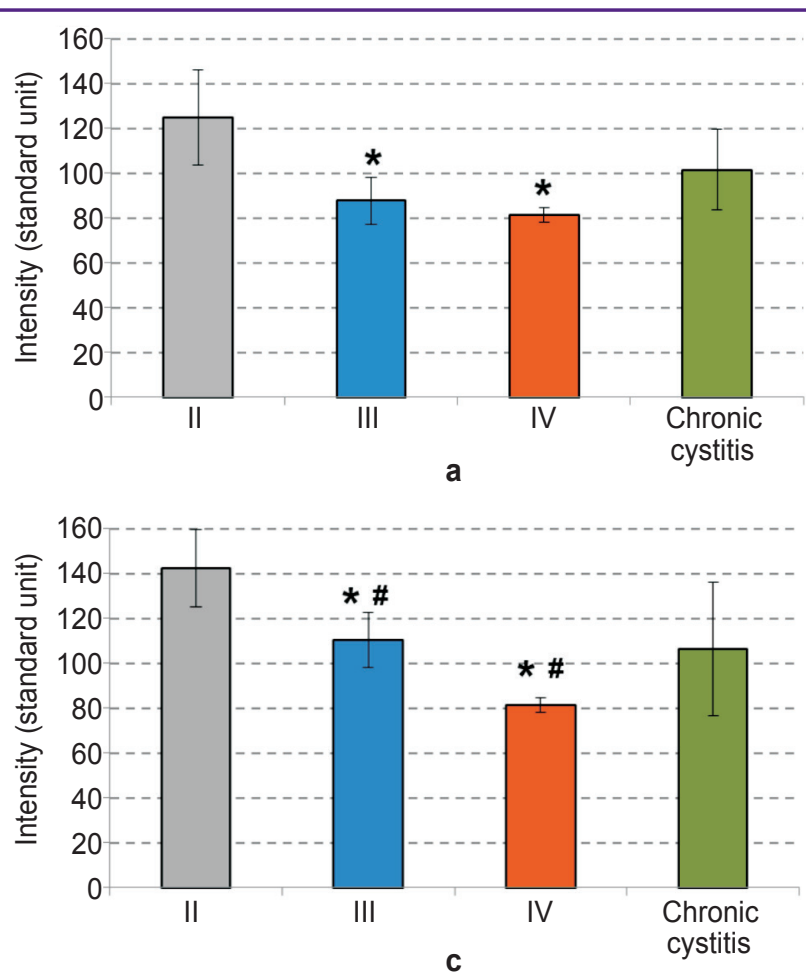
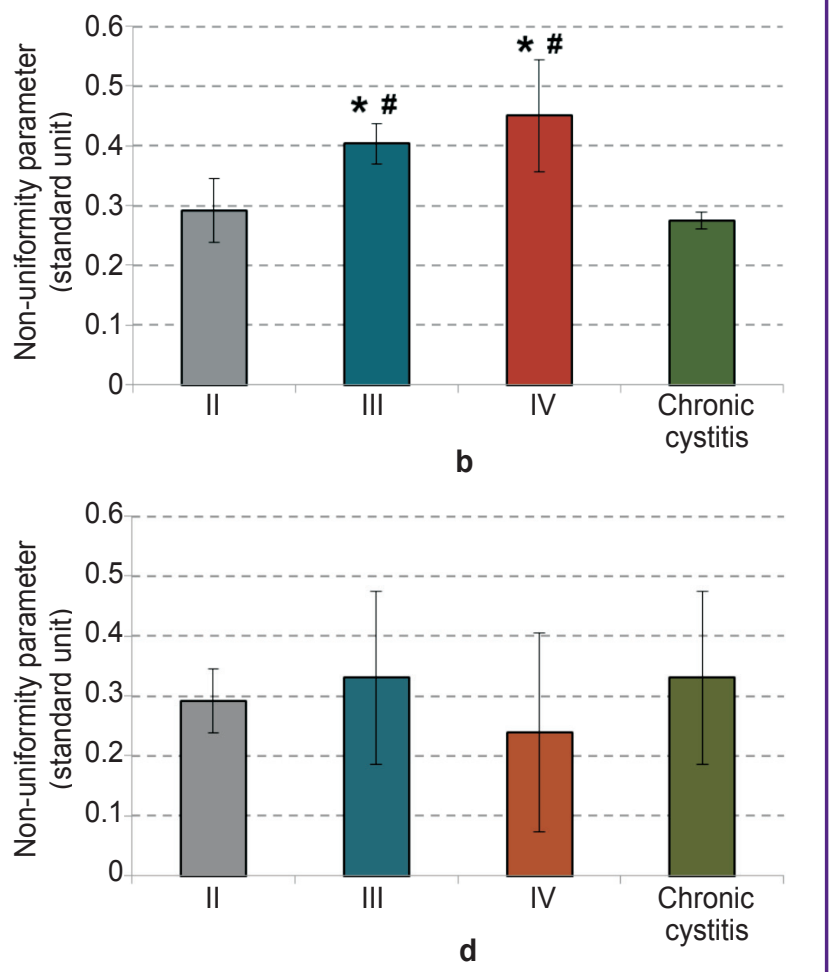

Figure 3. Results of the quantitative assessment of the collagen (a), (b) and elastin (c), (d) state obtained using nonlinear microscopy images depending on the severity of radiation complications:

(a) mean intensity of the collagen SHG signal dispersion; (b) non-uniformity parameter of the collagen SHG signal dispersion;

(c) mean intensity of the elastin TPEF signal dispersion; (d) non-uniformity parameter of the elastin TPEF signal dispersion; * statistically significant differences between the groups with severe radiation damages (grade III, IV) and grade II, p $\leq 0.05$;

\# between the groups of radiation damage and chronic cystitis, $p \leq 0.05$ 
damage, the mean intensity values of SHG and TPEF signals are lower than in severity grade II, in chronic cystitis the values of this parameter are at the level of grades II-III. Such reduction of these values reflects a marked disorganization (fragmentation) of the fibers relative to their native structure that results in the lower ability of the fibers to generate SHG or TPEF signals. Increase in the values of SHG signal non-uniformity parameter in the bladder radiation damage grades III and IV in comparison with grade II and chronic cystitis reflects the same tendency.

\section{Discussion}

One of the scientific achievements in the medicine of the XXI century was a new viewpoint regarding the importance of the connective tissue in the body as an integrating global network [20, 21]. A number of works show a significant role of the connective tissue which is involved in any pathological processes occurring in the organs [22, 23] including those induced by a radiation exposure [24].

The role of the extracellular matrix in the physiological processes of the tissues is fundamental since a fibrous component (mainly collagen and elastin fibers) and an amorphous component (glycoproteins, glycosaminoglycans, proteoglycans, and their agents) are known to be biochemically and functionally connected. Collagen and elastin fibers play an important role in the regulation of tissue metabolism being supporting structures and chemical stimulants for the cells [20]. Destruction of the collagen fibers of any origin is of great significance for the pathogenesis of the urinary bladder damage and often causes subsequent functional disorders. Therefore, knowledge about the initial state of the bladder connective tissue matrix prior to radiotherapy and about structural changes originated after it is very important for the correction of the complications developed and maintenance of the bladder functional reserves.

Traditional use of the standard methods of histological investigation was unable to provide full information on the state of the leading component of tissue metabolism in the bladder wall, i.e. extracellular matrix. Nonlinear microscopy made it possible to perform qualitative and quantitative assessment of the connective tissue matrix in clinically different severity grades of radiation therapy complications.

The use of nonlinear optical microscopy, as well as the application of computer-aided processing of the acquired digital stroma images in the SHG and TPEF modes, permitted us to quantitatively analyze the state of the bladder collagen and elastin in complications caused by the radiation therapy of the female reproductive system tumors.

Evaluation of the state of the bladder connective tissue matrix prior to radiation exposure provides the possibility to identify a group of risk for the development of heavy radiation damages. Besides, the results obtained can serve as a pathogenetic base for designing non-invasive optical tools of monitoring radiation-induced damages in clinical practice. Cross-polarizing OCT method is likely to become the basic one, the potential of which has been previously estimated by our research group in a number of investigations $[12,15,25]$ to be a minimally invasive real time technique of the objective control.

\section{Conclusion}

A nonlinear microscopy method makes it possible to assess both qualitatively and quantitatively spatial and structural organization of the extracellular bladder matrix in radiation damage and chronic cystitis, to determine the severity degree of complications in radiation damage, and to predict their course.

Study funding. The work was supported by the Russian Foundation for Basic Research, grant No.1607-00655.

Conflicts of interest. The authors have no conflicts of interest to declare.

\section{References}

1. Dörr W. Radiation effect in normal tissue - principles of damage and protection. Nuklearmedizin 2010; 49(Suppl 1): S53-S58.

2. Dörr W., Hendry J.H. Consequential late effects in normal tissues. Radiother Oncol 2001; 61(3): 223-231, https:// doi.org/10.1016/s0167-8140(01)00429-7.

3. Richie J.P. Onkourologiya [Oncourology]. Moscow: Izdatel'stvo BINOM; 2011; 896 p.

4. Denham J.W., Hauer-Jensen M. The radiotherapeutic injury - a complex 'wound'. Radiother Oncol 2002; 63(2): 129-145, https://doi.org/10.1016/s0167-8140(02)00060-9.

5. Baker D.G., Krochak R.J. The response of the microvascular system to radiation: a review. Cancer Invest 1989; 7(3): 287-294, https://doi. org/10.3109/07357908909039849.

6. Yarnold J., Brotons M.C. Pathogenetic mechanisms in radiation fibrosis. Radiother Oncol 2010; 97(1): 149-161, https://doi.org/10.1016/j.radonc.2010.09.002.

7. Jaal J., Dörr W. Radiation effects on cellularity, proliferation and EGFR expression in mouse bladder urothelium. Radiat Res 2010; 173(4): 479-485, https://doi. org/10.1667/rr1759.1.

8. Fiorino C., Rancati T., Valdagni R. Predictive models of toxicity in external radiotherapy: dosimetric issues. Cancer 2009; 115(13 Suppl): 3135-3140, https://doi.org/10.1002/cncr.24354.

9. Cancer Therapy Evaluation Program, Common Terminology Criteria for Adverse Events (CTCAE) Version 4.0. 2009.

10. Gubarkova E.V., Dudenkova V.V., Feldchtein F.I., Timofeeva L.B., Kiseleva E.B., Kuznetsov S.S., Shakhov B.E., Moiseev A.A., Gelikonov V.M., Gelikonov G.V., Vitkin A., Gladkova N.D. Multi-modal optical imaging characterization of atherosclerotic plaques. J Biophotonics 2015; 9(10): 10091020, https://doi.org/10.1002/jbio.201500223.

11. Jain M., Robinson B.D., Scherr D.S., Sterling J., 
Lee M.M., Wysock J., Rubin M.A., Maxfield F.R., Zipfel W.R., Webb W.W., Mukherjee S. Multiphoton microscopy in the evaluation of human bladder biopsies. Arch Pathol Lab Med 2012; 136(5): 517-526, https://doi.org/10.5858/arpa.20110147-oa.

12. Kiseleva E., Kirillin M., Feldchtein F., Vitkin A., Sergeeva E., Zagaynova E., Streltzova O., Shakhov B., Gubarkova E., Gladkova N. Differential diagnosis of human bladder mucosa pathologies in vivo with cross-polarization optical coherence tomography. Biomed Opt Express 2015; 6(4): 1464-1476, https://doi.org/10.1364/boe.6.001464.

13. Yasui T., Tanaka R., Hase E., Fukushima S., Araki T. In vivo time-lapse imaging of skin burn wound healing using second-harmonic generation microscopy. In: Periasamy A., So P.T.C., König K. (editors). Multiphoton Microscopy in the Biomedical Sciences XIV. SPIE; 2014, https://doi. org/10.1117/12.2038022.

14. Maslennikova A., Kochueva M., Ignatieva N., Vitkin A., Zakharkina O., Kamensky V., Sergeeva E., Kiseleva E., Bagratashvili V. Effects of gamma irradiation on collagen damage and remodeling. Int J Radiat Biol 2015; 91(3): 240247, https://doi.org/10.3109/09553002.2014.969848.

15. Streltsova O.S., Maslennikova A.V., Yunusova K.E., Dudenkova V.V., Kiseleva E.B., Kochueva M.V., Tararova E.A., Malikov D.K., Vorobieva A.S., Krupin V.N. Nonlinear microscopy in studying extracellular matrix state of the urinary bladder in severe complications after radiation therapy of female pelvic tumors. Sovremennye tehnologii $v$ medicine 2017; 9(2): 19, https://doi.org/10.17691/stm2017.9.2.02.

16. Rasband W.S. Image J: Image Processing and Analysis in Java. U.S. National Institutes of Health, Bethesda, Maryland, USA; 1997-2016. URL: http://rsb.info.nih.gov/ij/.

17. Balu M., Kelly K.M., Zachary C.B., Harris R.M., Krasieva T.B., König K., Durkin A.J., Tromberg B.J. Distinguishing between benign and malignant melanocytic nevi by in vivo multiphoton microscopy. Cancer Res 2014; 74(10):
2688-2697, https://doi.org/10.1158/0008-5472.can-13-2582.

18. Adur J., Pelegati V.B., Costa L.F., Pietro L., de Thomaz A.A., Almeida D.B., Bottcher-Luiz F., Andrade L.A., Cesar C.L. Recognition of serous ovarian tumors in human samples by multimodal nonlinear optical microscopy. J Biomed Opt 2011; 16(9): 096017, https://doi.org/10.1117/1.3626575.

19. Zhao W., Robbins M. Inflammation and chronic oxidative stress in radiation-induced late normal tissue injury: therapeutic implications. Curr Med Chem 2009; 16(2): 130 143, https://doi.org/10.2174/092986709787002790.

20. Langevin H.M. Connective tissue: a body-wide signaling network? Med Hypotheses 2006; 66(6): 1074-1077, https:// doi.org/10.1016/j.mehy.2005.12.032.

21. Stecco C. Functional atlas of the human fascial system. Elsevier; 2015.

22. Gladkova N., Kiseleva E., Streltsova O., Prodanets N., Snopova L., Karabut M., Gubarkova E., Zagaynova E. Combined use of fluorescence cystoscopy and cross-polarization OCT for diagnosis of bladder cancer and correlation with immunohistochemical markers. J Biophotonics 2013; 6(9): 687-698, https://doi.org/10.1002/ jbio.201200105.

23. Ganganna K., Shetty P., Shroff S.E. Collagen in histologic stages of oral submucous fibrosis: a polarizing microscopic study. J Oral Maxillofac Pathol 2012; 16(2): 162166, https://doi.org/10.4103/0973-029x.98446.

24. Hölscher T., Bentzen S.M., Baumann M. Influence of connective tissue diseases on the expression of radiation side effects: a systematic review. Radiother Oncol 2006; 78(2): 123-130, https://doi.org/10.1016/j.radonc.2005.12.013.

25. Kiseleva E., Gladkova N., Streltzova O., Kirillin M., Maslennikova A., Dudenkova V., Yunusova K., Sergeeva E. Cross-polarization OCT for in vivo diagnostics and prediction of bladder cancer. In: Bladder cancer - management of NMI and muscle-invasive cancer. InTech; 2017; 43-61, https://doi. org/10.5772/67473. 